\title{
Kernos
}

Revue internationale et pluridisciplinaire de religion grecque antique

$25 \mid 2012$

Varia

\section{Haralampos V. HARISSIS, Anastasios V. HARISSIS, Apiculture in the Prehistoric Aegean. Minoan and Mycenaean Symbols Revisited}

\author{
Ariadni Gartziou-Tatti
}

\section{(2) OpenEdition}

Journals

Édition électronique

URL : http://journals.openedition.org/kernos/2046

DOI : 10.4000/kernos.2046

ISSN : 2034-7871

Éditeur

Centre international d'étude de la religion grecque antique

Édition imprimée

Date de publication : 26 octobre 2012

Pagination : 334-335

ISSN : 0776-3824

Référence électronique

Ariadni Gartziou-Tatti, « Haralampos V. HARISSIS, Anastasios V. HARISSIS, Apiculture in the Prehistoric Aegean. Minoan and Mycenaean Symbols Revisited », Kernos [En ligne], 25 | 2012, mis en ligne le 01 octobre 2012, consulté le 21 septembre 2020. URL : http://journals.openedition.org/kernos/2046 ; DOI : https://doi.org/10.4000/kernos.2046

Ce document a été généré automatiquement le 21 septembre 2020.

Kernos 


\title{
Haralampos V. HARISSIS, Anastasios V. HARISSIS, Apiculture in the Prehistoric Aegean. Minoan and Mycenaean Symbols Revisited
}

\author{
Ariadni Gartziou-Tatti
}

\section{RÉFÉRENCE}

Haralampos V. HARISSIS, Anastasios V. HARISSIS, Apiculture in the Prehistoric Aegean. Minoan and Mycenaean Symbols Revisited, Oxford, British Archaeological Reports, 2009. 1 vol. $21 \times$ 29,7 cm, 95 p. (BAR International Series,1958). ISBN : 978-1-4073-0454-0.

1 La publication, presque simultanée mais indépendante, ces dernières années, de trois études portant sur l'abeille et l'apiculture démontre un intérêt scientifique important pour la place centrale qu'occupe l'abeille dans la vie économique, politique et religieuse des Grecs anciens ${ }^{1}$. Parmi ces études, celle de Haralampos et Anastasios V. Harissis se concentre sur la présentation des témoignages relatifs au monde de l'abeille dans l'Égée préhistorique. Fidèles à l'approche historique, les $\mathrm{A}$. cherchent à répondre d'abord à la question de la connaissance des pratiques d'apiculture dans le monde minoen et mycénien et, par la suite, accentuent les éléments qui confirment leur hypothèse. Dans la $1^{\text {re }}$ partie (p. 3-37), après une brève synthèse récapitulative des pratiques apicoles connues en Orient, le rappel des récits mythologiques (comme celui des Courètes) et la présentation des témoignages littéraires ultérieurs, les A. mettent en lumière l'usage et la connaissance des pratiques similaires dans la civilisation minoenne. Grace à l'analyse des trouvailles archéologiques, ils arrivent à la conclusion que le monde minoen et mycénien utilisaient des ruches similaires aux ruches traditionnelles en pierre, en céramique ou tressées, des enfumoirs et d'autres pièces apicoles. Ce constat les conduit à une révision radicale des interprétations concernant la nature et l'utilisation des 
célèbres trouvailles de la petite chambre de Knossos, trouvailles qui, d'après eux, n'étaient que des outils apicoles, c'est-à-dire des enfumoirs ou des pots d'extraction du miel, de la cire et autre.

Dans la $2^{\mathrm{e}}$ partie (p. 39-72), les A.intègrent l'apiculture dans le cadre des activités économiques et sociales du monde de l'Égée préhistorique et ils illustrent ainsi l'importance de la pratique apicole. Ils s'écartent totalement des schémas connus, aussi bien anthropologiques que religieux, formés par Evans et par ceux qui l'ont suivi. Les discussions théoriques qui portent sur l'interprétation des symboles cultuels, sur l'existence d'une ou de plusieurs divinités minoennes, sur les lieux et la nature du culte, sur l'impact de l'Orient dans la formation des conceptions religieuses, etc., ont préoccupé pendant longtemps les spécialistes de la civilisation minoenne, sans qu'aucune théorie ne fasse l'unanimité. La thématique qui concerne la présence de la divinité féminine ${ }^{2}$, le rôle de la double hache ${ }^{3}$, les cornes de consécration ${ }^{4}$, la présence d'oiseaux $^{5}$, de bétyles ${ }^{6}$, les pratiques sacrificielles ${ }^{7}$ et le rôle des autels a été plusieurs fois analysée et sous des angles différents; bon nombre d'éléments restent cependant encore dans l'ombre.

3 Les A. refusent les interprétations généralisantes des sujets en question et ils proposent une approche aussi intéressante que provocatrice. D'après eux, la lecture des représentations, surtout celles de certains célèbres anneaux en or, exige une nouvelle analyse. Il ne s'agit pas de données cultuelles mais d'objets relatifs au monde de l'apiculture. Ils soutiennent d'ailleurs que, sur la représentation de l'arbre sacré des anneaux, se posent des essaims d'abeilles (p.43), que les points que l'on voit - des perles aux mains et autour du cou des femmes - sont des représentations d'abeilles (p. 46), et que les oiseaux qui apparaissent sont des merops (p. 50). Les femmes qui ont été considérées comme les représentations de la Grande Déesse pourraient être des apicultrices (p. $51 \mathrm{sq}$.), les figures des bétyles pourraient également renvoyer aux ruches (p. $53 \mathrm{sq}$.), les petites figures masculines ne sont pas de «dieux volants », mais des personnages qui se trouvent en arrière des personnages du premier plan et tiennent entre leurs mains des boucliers en cuivre, position qui renvoie à une technique très connue d'immobilisation d'essaims par les apiculteurs. Ils soutiennent aussi que ce qui est considéré, à cause de sa forme, comme un autel, pourrait très bien représenter un ustensile d'apiculture (p. 57 sq.), que les cornes de consécration sont en rapport avec les cérémonies apotropaïques magiques pour la protection des ruches (p. 62 sq.) et que la double hache n'est que la représentation schématique de la figure de l'abeille (p. 68 sq.). La pratique globale s'intègre dans le cadre de l'économie du palais ; ces scènes d'apiculture sur les anneaux et sur les sceaux appartenaient aux titulaires responsables du scellement, de l'empaquetage et de la diffusion des produits apicoles.

Malgré quelques faiblesses méthodologiques, les fautes d'impression et la forme très concise de la présentation (dont il faut néanmoins souligner la connaissance de la bibliographie et des pratiques apicoles populaires et traditionnelles), cette étude, étonnamment originale, peut ouvrir de nouveau le dialogue sur l'interprétation des trouvailles archéologiques. Même si la question de la double hache et celle des cornes de consécration demandent une analyse plus approfondie, la discussion, débarrassée de préjugés et d'obsessions pour des schémas théoriques, doit être continuée sur la base d'une analyse approfondie de toutes les données archéologiques, de représentations iconographiques, d'informations provenant de la lecture des tablettes en écriture linéaire B tout comme de la comparaison avec le monde oriental. Toute approche 
d'interprétation univoque immobilise non seulement l'optique de l'approche en question mais aussi l'ensemble des éléments des fouilles et des trouvailles archéologiques liés au cycle rituel et religieux du monde minoen. La lecture du monde du miel peut éclairer la vie sociale et économique de l'Égée préhistorique dans son ensemble, où une place importante est occupée par l'étude d'autres activités, relatives par exemple au monde du vin, de l'huile, du safran ${ }^{8}$, etc.

\section{NOTES}

1. Voir R. BORTOLIn, Archeologia del miele, Alessandria, 2008; M. GIUMAN, MELISSA. Archeologia delle ape e del miel nella grecia antica, Roma, 2008.

2. Voir les approches differentes de D. RUBIN, The Development of Scholarly Thinking on Minoan Religion from Sir Arthur Evans to the Present, MA, University of Concordia, 1993 ; R. LAFFINEUR, R.HÄGG (éds), POTNIA. Deities and Religion in the Aegean Bronge Age, Liège, 2001 (Aegeum, 22); M.L. MOss, The Minoan Pantheon: Towards an Understanging of its Nature and Extent, Oxford, 2005(BAR International Series, 1343) ; G.C. GESELL, "From Knossos to Kavoussi: The Popularizing of the Minoan Palace Goddess ", in XAPIL: Essays in Honor of Sara A. Immerwarh, Princeton, 2004 (Hesperia, Suppl. 33), p. 131-150 ; J. CROWLEY, «In Honour of the Gods - but which Gods? Identifying Deities in Aegean Glyptic ", in L.A. HITCHCOCKH, R. LAFFINEUR, J.CROWLEY (éds), DAIS. The Aegean Feast, Liège, 2008 (Aegaeum,29), p. 75-87 ; N. MARINATOS, Minoan Religion. Ritual, Image and Symbol, Columbia, 1993 ; Minoan Kingship and the Solar Goddess: A Near Eastern Koine, University of Illinois Press, 2010; F. BLAKOLMER, «A Pantheon without Attributes? Goddesses and Gods in Minoan and Mycenaean Iconography ", in J. MYLONOPOUlos (éd.), Divine Images and Human Imaginations in Ancient Greece and Rome, Leiden, 2010, p. 21-61.

3. Moss, The Minoan Pantheon, p. 17, 197 sq. ; M. HAYSOM, « The Double-axe: A Contextual Approach to the Understanding of a Cretan Symbol in the Neopalatial Period ", Oxford Journal of Archeology 29 (2010), p. 35-55 ; MARINATOS, Minoan Religion, p. 120 sq.

4. A.L. D'AGATA, «Late Minoan Crete and Horns of Consecrations: A Symbol in Action », in J.L. CROWLEY, R. LAFFINEUR (éd.), EIKON. Aegean Bronge Age Iconography: Shaping a Methodology, Liège, 1992(Aegeum,8), p. 247-255 ; moss, The Minoan Pantheon, p. 198 sq., 210 sq.; E. BANOU, " Minoan "Horns of Consecration" Revised: A Symbol of Sun Worship in Palatial and Post-palatial Crete? ", Mediterannean Archaeology and Archaeometry 8.1 (2008), p. 27-47 ; MARINATOS, Minoan Religion,p. 67 sq., $105 \mathrm{sq}$.

5. Moss, The Minoan Pantheon, p. 13 et passim.

6. E. KYRIAKIDIS, «Pithos or Baetyl? On The Interpretation of a Group of Minoan Rings ", OAth 25-26 (2000-2001), p. 117-118; Moss, The Minoan Pantheon, p. 195; V. LA ROSA, « Minoan Baityl: Between Funerary Rituals and Epiphany ", in POTNIA (supra),p. 221-227.

7. N. MARINATOS, Minoan Sacrifice Ritual. Cult Practices and Symbolism, Stockholm, 1986; M. GUGGISBERG, "Animal Figures and Sacrificial Rituals at the End of the Bronze Age ", in A.-L. SCHALLIN (éd.), en collaboration avec P. PAKKANEN, Encounters with Mycenaean Figures and Figurines. Papers presented at a Seminar at the Swedish Institute at Athens, 27-29 April 2001, Stockholm, Svenska Institutet i Athen, 2009 (ActaAth in-8 $\left.{ }^{\circ}, 20\right)$, p. 125-138. 
8. J. DAY, "Crocuses in Context. A Diachronic Survey of the Crocus Motif in the Aegean Age", Hesperia 28 (2011), p. 337-379.

\section{AUTEURS}

ARIADNI GARTZIOU-TATTI

Université de Ioannina 